\title{
Frictional resistance of self-ligating versus conventional brackets in different bracket- archwire-angle combinations
}

\author{
Maria Regina Guerra MONTEIRO1', Licinio Esmeraldo da SILVA², Carlos Nelson ELIAS ${ }^{3}$, Oswaldo de Vasconcellos \\ VILELLA $^{4}$ \\ 1- Department of Orthodontics, Fluminense Federal University, Niteroi, RJ, Brazil; private practice, Rio de Janeiro, RJ, Brazil. \\ 2- Department of Statistics, Fluminense Federal University, Niterói, RJ, Brazil. \\ 3- Materials Science Department, Military Engineering Institute, Rio de Janeiro, Brazil. \\ 4- Department of Orthodontics, Fluminense Federal University, Niterói, RJ, Brazil.
}

Corresponding address: Maria Regina Guerra Monteiro - Estrada Francisco da Cruz Nunes - 6501/sl 310 - Itaipu - Niterói - RJ - 24350-010 - Brazil - e-mail: mreginaguerra@yahoo.com.br

Submitted: November 24, 2013 - Modification: January 27, 2014 - Accepted: March 2, 2014

\section{ABSTRACT}

\begin{abstract}
$\mathrm{O}$ bjective: To compare the influence of archwire material ( $\mathrm{NiTi}$, beta-Ti and stainless steel) and brackets design (self-ligating and conventional) on the frictional force resistance. Material and Methods: Two types of brackets (self-ligating brackets - Smartclip, 3M/Unitek - and conventional brackets - Gemini, 3M/Unitek) with three (0, 5, and 10 degrees) slot angulation attached with elastomeric ligatures (TP Orthodontics) were tested. All brackets were tested with archwire $0.019 " \times 0.025^{\prime \prime}$ nickel-titanium, beta-titanium, and stainless steel (Unitek/3M). The mechanical testing was performed with a universal testing machine EMIC DL 10000 (EMIC Co, Brazil). The wires were pulled from the bracket slots at a cross-head speed of $3 \mathrm{~mm} / \mathrm{min}$ until $2 \mathrm{~mm}$ displacement. Results: Self-ligating brackets produced significantly lower friction values compared with those of conventional brackets. Frictional force resistance values were directly proportional to the increase in the bracket/ wire angulation. With regard to conventional brackets, stainless steel wires had the lowest friction force values, followed by nickel-titanium and beta-titanium ones. With regard to self-ligating brackets, the nickel-titanium wires had the lowest friction values, significantly lower than those of other materials. Conclusion: Even at different angulations, the selfligating brackets showed significantly lower friction force values than the conventional brackets. Combined with nickel-titanium wires, the self-ligating brackets exhibit much lower friction, possibly due to the contact between nickel-titanium clips and wires of the same material.
\end{abstract}

Keywords: Friction. Orthodontic brackets. Orthodontic wires. Biomaterials.

\section{INTRODUCTION}

Tooth movement can occur when the applied forces adequately overcome the friction at the bracket slot-archwire interface. High levels of frictional force between the bracket slot and the archwire might cause binding between the two components; this in turn result in little or no tooth movement ${ }^{18}$. Furthermore, low forces are considered desirable to conserve anchorage ${ }^{15}$ and reduce the risk of root resorption ${ }^{11}$. Therefore, it is essential to understand the friction between the bracket and the archwire so that the proper force can be applied to obtain adequate tooth movement and optimum biologic response ${ }^{21}$. Friction has been attributed to many factors, such as bracket type ${ }^{5,13}$, wire size and alloy ${ }^{14,15}$, method of ligation ${ }^{9,17}$ and angulation between the bracket slot surface and orthodontic wires ${ }^{25}$.

Recently, there has been increased use of the selfligating bracket. These brackets are characterized by having a "ligature" similar to an opening and closing cover or precision latch, thus avoiding the use of additional ligatures for attachment ${ }^{10}$. The 
main advantage of self-ligating brackets is the reduction of frictional forces. Studies comparing self-ligating brackets with different ligating systems for conventional brackets have reported and showed that the former has a significantly lower level of frictional resistance $1,4,6-9,17,18,20,27$. However, some studies that evaluated rectangular wires ${ }^{12,13,23,28}$ and applied moments ${ }^{22,23}$ showed no differences between self-ligating and conventional brackets $12,13,22,28$. Some authors still found a greater friction in the self-ligating brackets ${ }^{25}$. A recent systematic review ${ }^{5}$ concluded that, in comparison with conventional brackets, self-ligating brackets maintain lower friction only when coupled with small round archwires. Sufficient evidence, however, has not been found to claim that the self-ligating produce lower friction with large rectangular wires in the presence of tipping and/or torque.

It should be taken into account that reduced friction force is interesting in the initial stages of orthodontic treatment and during retraction of anterior teeth using sliding mechanics ${ }^{15}$. In the last phases, however, higher friction force can be used to obtain a three-dimensional control of the tooth position. The ideal self-ligating bracket is the one that can be conventionally attached when high friction is needed ${ }^{26}$. The design of four double wings of the Smartclip bracket allows ligatures to be used when friction is desired. To our knowledge, there are no studies in the literature evaluating friction in these brackets associated with different types of wire at different angles.

The objective of the present work was to analyze the influence of self-ligating brackets (Smartclip) combined with rectangular archwires even at different angulations on frictional forces and compare them with conventional brackets.

\section{MATERIAL AND METHODS}

Two types of brackets were tested: selfligating brackets (Smartclip, 3M Unitek, Monrovia, California, USA) and conventional brackets (Gemini, 3M Unitek, Monrovia, California, USA). Only upper central incisor brackets with $0.022 \times 0.028$-in slots and MBT prescription were used. The brackets were tested with $0.019^{\prime \prime} \times 0.025^{\prime \prime}$ nickel-titanium, betatitanium, and stainless steel archwires (3M Unitek, Monrovia, California, USA). Conventional brackets were attached by using elastomeric ligatures (TP Orthodontics, La Porte, IN, USA).

The dislocation resistance of the archwires resulted from friction force produced by the contact between the bracket slot surface and orthodontic wires segments of $5.0 \mathrm{~cm}$. Each bracket/wire combination was submitted to mechanical traction test at angulations of 0,5 , and 10 degrees. Each combination of bracket/wire/angulation was submitted to 10 tests by a single operator, thus totalizing 90 mechanical testing for each group of brackets and 180 for both groups.

The friction mechanical testing was performed by using a universal testing machine (EMIC DL 10000 , São José dos Pinhais, PR, Brazil) with load cell of $20 \mathrm{~N}$. For conducting such an experiment, devices were developed specially for this type of mechanical testing (Figures 1A, 1B, and 1C). Each wire segment was attached to a device containing a regulating inner hole. The brackets were positioned at the center of a circular piece and then fixed there
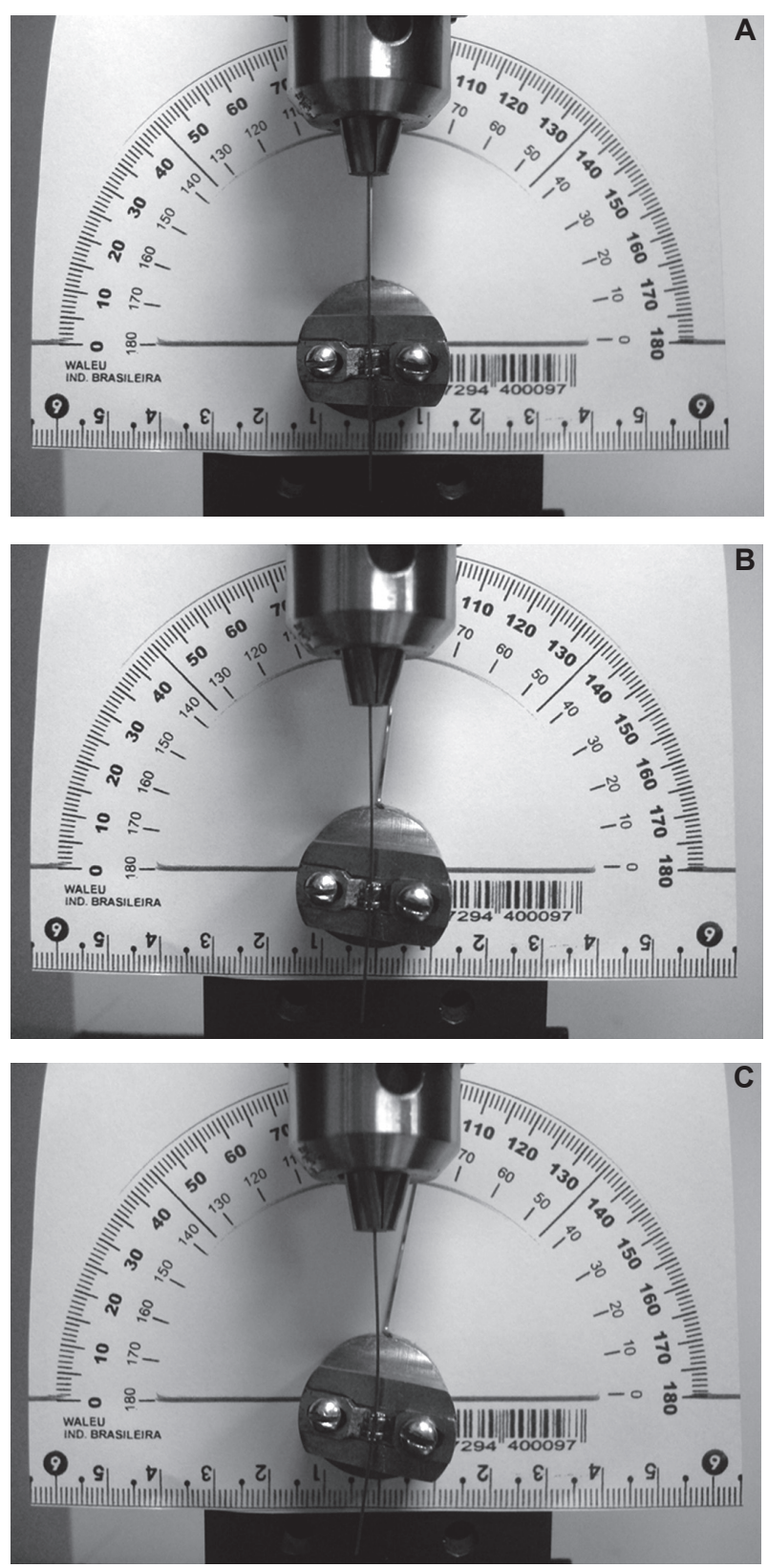

Figure 1- Setup for surface friction testing. A) Angulation of $0^{\circ}$ between bracket and wire; B) angulation of $5^{\circ}$ between bracket and wire; and $\mathrm{C}$ ) angulation of $10^{\circ}$ between bracket and wire 
with screws located at the tip of this piece. As the brackets had MBT prescription (170 torque), the circular piece had an angulated shape in order to eliminate the angulation of the brackets.

Before the mechanical testing, both wires and brackets were cleaned with alcohol ${ }^{9}$. For the conventional brackets, elastomeric ligatures were used to attach the wires. Following placement of each ligature, a 3-minute period was waited to allow them to relax ${ }^{12,13,18,28}$. The ligatures were installed by means of an applicator (Morelli 75.01.002, Sorocaba, SP, Brazil) and replaced before every test. For self-ligating brackets, no type of attachment was used.

For the mechanical testing of brackets with zero-degree angulation, the wire segments were positioned perpendicular to the base of the circular piece, thus keeping a passive contact with the bracket slots. At angulations of 5 and 10 degrees, the circular piece was turned in relation to the long axis of the orthodontic wires.

The wires were pulled through the bracket slots at a cross-head speed of $3 \mathrm{~mm} / \mathrm{min}$ until a displacement of $2 \mathrm{~mm}$. The friction was measured under dry conditions and at room temperature $\left(20^{\circ} \mathrm{C} \pm 2^{\circ} \mathrm{C}\right)$. The values of maximum force ( $\mathrm{gf}$ ) produced were recorded by the TESC software, version 3.04, and then statistically analyzed. Arithmetic means, standard deviations, inter- quartile ranges, and minimum and maximum values were calculated for combinations of bracket/wire/ angulation. Kruskal-Wallis test was applied for study of the effect of wire type and angulation on frictional resistance, whereas Mann-Whitney test was used for post-hoc analysis.

A scanning electron microscopy (SEM-JEOL JSM 5800 LV, Tokyo, Japan) was used to analyze the surface morphology of both bracket and archwire.

\section{RESULTS}

The following variables were examined to determine the static friction: type of attachment, type of wire, and angulation between archwire and bracket slot. The influence of type of wire material and angulation on friction was analyzed separately for conventional and self-ligating brackets, thus allowing the behavior of these variables to be assessed in relation to both groups of brackets. Descriptive statistics for different combinations of bracket/wire/angulation is shown in Table 1.

Kruskal-Wallis test showed a significant effect of the type of wire material $(p<0.0001)$. With regard to conventional brackets, Mann-Whitney test showed statistic differences in the maximum friction force produced by stainless steel, nickeltitanium $(p=0.011)$, and beta-titanium $(p<0.0001)$ archwires, although nickel-titanium wires presented

Table 1- Statistical description of maximum forces for different variables regarding conventional and self-ligating brackets

\begin{tabular}{|c|c|c|c|c|c|c|c|c|}
\hline & variables & $\mathbf{n}$ & $\min$ & $\max$ & Mean & SD & median & iqr \\
\hline Self-ligating & Wire Type & & & & & & & \\
\hline \multirow[t]{3}{*}{ Brackets } & Stainless steel & 30 & 0.2 & 245 & 105.1 & 101.95 & 75.5 & 238.86 \\
\hline & Nickel-Titanium & 30 & 0.6 & 62.7 & 27.5 & 24.6 & 22.5 & 57.51 \\
\hline & Beta-Titanium & 30 & 0.8 & 308.8 & 134.2 & 116.98 & 120.6 & 272.4 \\
\hline Conventional & Wire Type & & & & & & & \\
\hline \multirow[t]{3}{*}{ Brackets } & Stainless steel & 30 & 106.5 & 247.4 & 166.9 & 55.91 & 140.1 & 124.98 \\
\hline & Nickel-Titanium & 30 & 138.4 & 283.8 & 185.4 & 57.7 & 148.2 & 109.58 \\
\hline & Beta-Titanium & 30 & 138.6 & 327 & 247.8 & 68.9 & 264.6 & 144.66 \\
\hline Self-ligating & Angulation & & & & & & & \\
\hline \multirow[t]{3}{*}{ Brackets } & 0 degree & 30 & 0.21 & 1.98 & 0.86 & 0.44 & 0.78 & 0.51 \\
\hline & 5 degrees & 30 & 20.2 & 130 & 72.5 & 40.98 & 75.5 & 90.32 \\
\hline & 10 degrees & 30 & 55.8 & 308.8 & 193.5 & 98.4 & 241.6 & 214.13 \\
\hline Conventional & Angulation & & & & & & & \\
\hline \multirow[t]{7}{*}{ Brackets } & 0 degree & 30 & 106.5 & 180.4 & 136.8 & 19.36 & 139.5 & 27.31 \\
\hline & 5 degrees & 30 & 137.6 & 275 & 185.9 & 56.21 & 150.8 & 120.46 \\
\hline & 10 degrees & 30 & 232.1 & 327 & 274.2 & 32.88 & 263.7 & 67.67 \\
\hline & Ligation Type & & & & & & & \\
\hline & Self-ligating & 90 & 0.2 & 308.8 & 89 & 100.47 & 58.9 & 128.27 \\
\hline & (Smartclip) & & & & & & & \\
\hline & Conventional (Gemini) & 90 & 106.5 & 327 & 199 & 69.06 & 163.5 & 121.3 \\
\hline
\end{tabular}



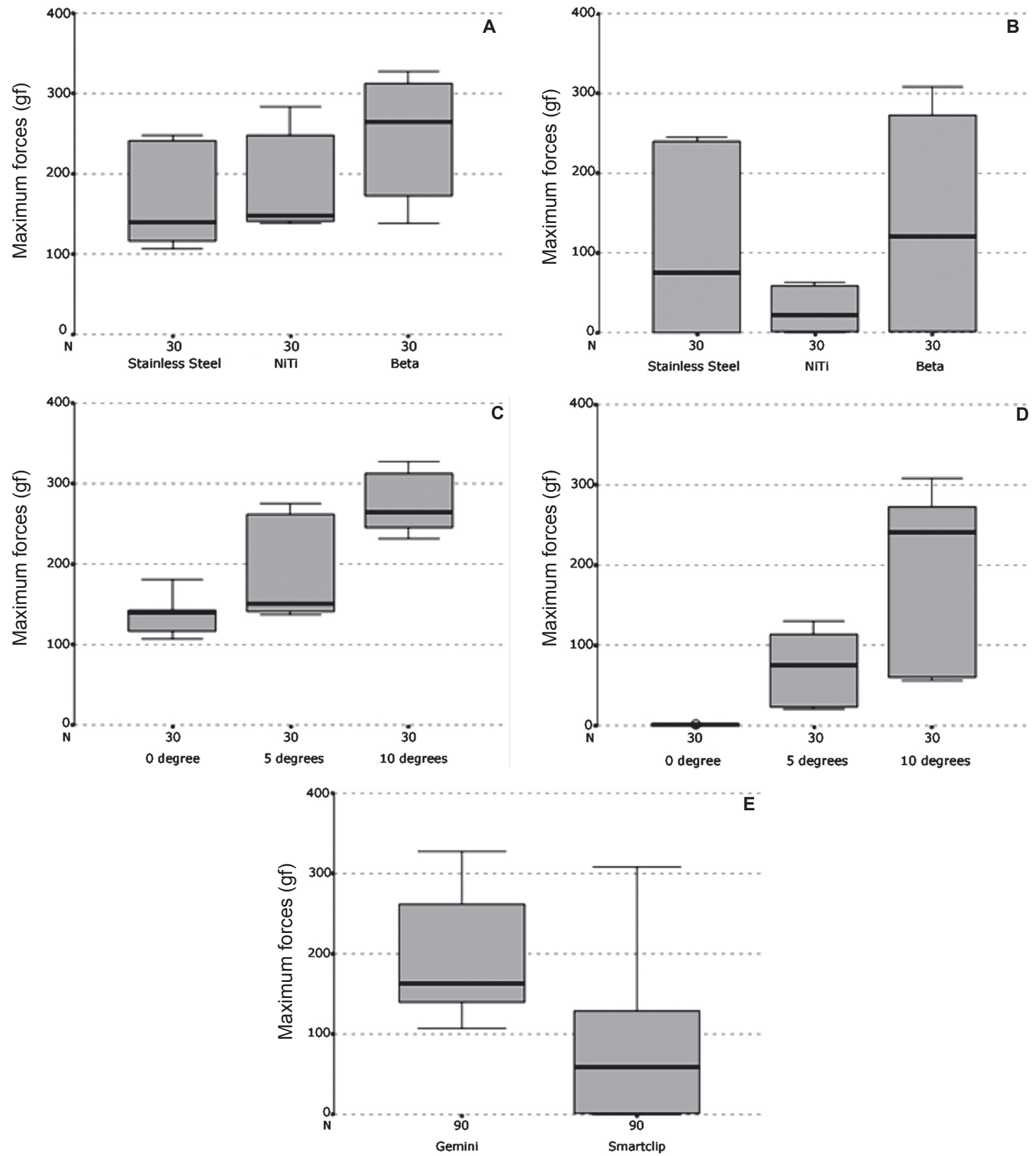

Figure 2- A) Maximum forces (gf) exerted on conventional brackets by type of orthodontic wire; B) Maximum forces (gf) exerted on self-ligating brackets by type of orthodontic wire; C) Maximum forces (gf) exerted on conventional brackets by angulation; D) Maximum forces (gf) exerted on self-ligating brackets by angulation; and E) Maximum forces (gf) exerted by ligation method

statistic difference compared to the beta-titanium one $(p=0.0003)$. By using the mean values, it was observed that lowest frictional forces were produced by stainless steel wire, followed by nickel-titanium and beta-titanium ones (Figure 2A). With regard to self-ligating brackets, the Kruskal-Wallis test showed that type of wire material had a significant effect $(p<0.0003)$. The Mann-Whitney test showed that the maximum force of stainless steel wire differed from that of the nickel-titanium wire $(p=0.013)$. The beta-titanium wire differed from the nickel-titanium one $(p=0.0002)$, but did not present difference compared to stainless steel wire $(p=0.028)$. The observation of mean values showed that nickel-titanium wire had produced the lowest forces, followed by stainless steel and beta-titanium 
archwires, without statistical differences between them (Figure 2B).

Kruskal-Wallis showed a significant effect of angulation $(p<0.0001)$. With regard to conventional brackets, the Mann-Whitney test indicated differences in the maximum force values produced by different angulations. It was observed that zero-degree angulation differed from fivedegree $(p=0.0002)$ and ten-degree $(p=0.0001)$ angulations, whereas five-degree angulation differed from the ten-degree one $(p<0.0002)$ (Figures 2C and 2D). Mean values showed that both conventional and self-ligating brackets produced lower frictional forces at angulations of $0^{\circ}$, followed by $5^{\circ}$ and $10^{\circ}$.

The Mann-Whitney test showed statistically significant difference $(p<0.0001)$ between maximum forces produced by attachment systems using conventional and self-ligating brackets, with the former (Gemini) having greater maximum force compared to the latter (Smartclip) (Figure 2E). The interactions between each type of bracket and the different archwires and angulations are shown in Figure 3.

\section{DISCUSSION}

In the present study, conventional and selfligating brackets were submitted to mechanical
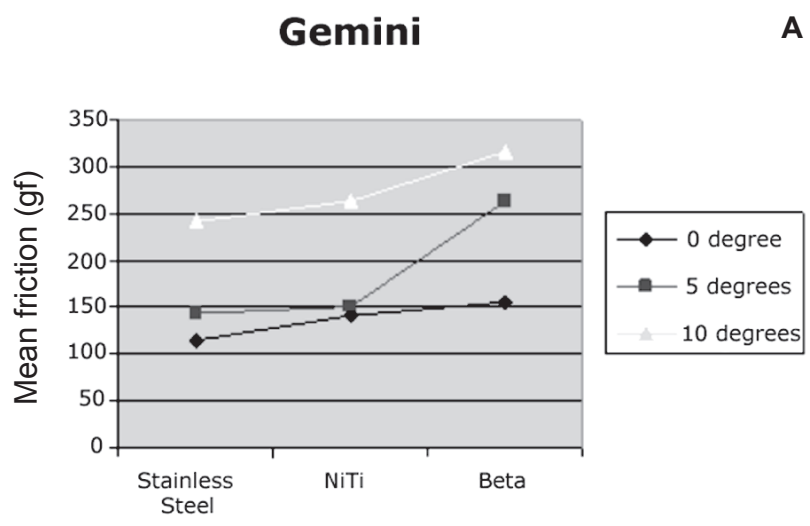

Smartclip

B

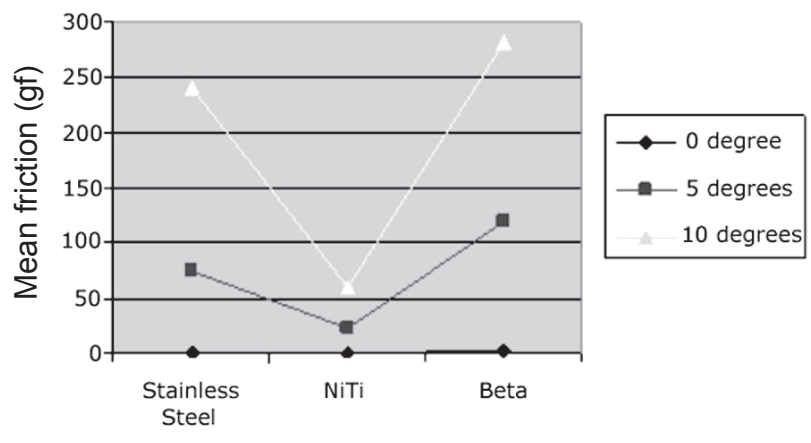

Figure 3- Mean friction values (gf) of interactions between brackets and types of orthodontic wires at $0^{\circ}, 5^{\circ}$ and $10^{\circ}$. A) Gemini; B) Smartclip
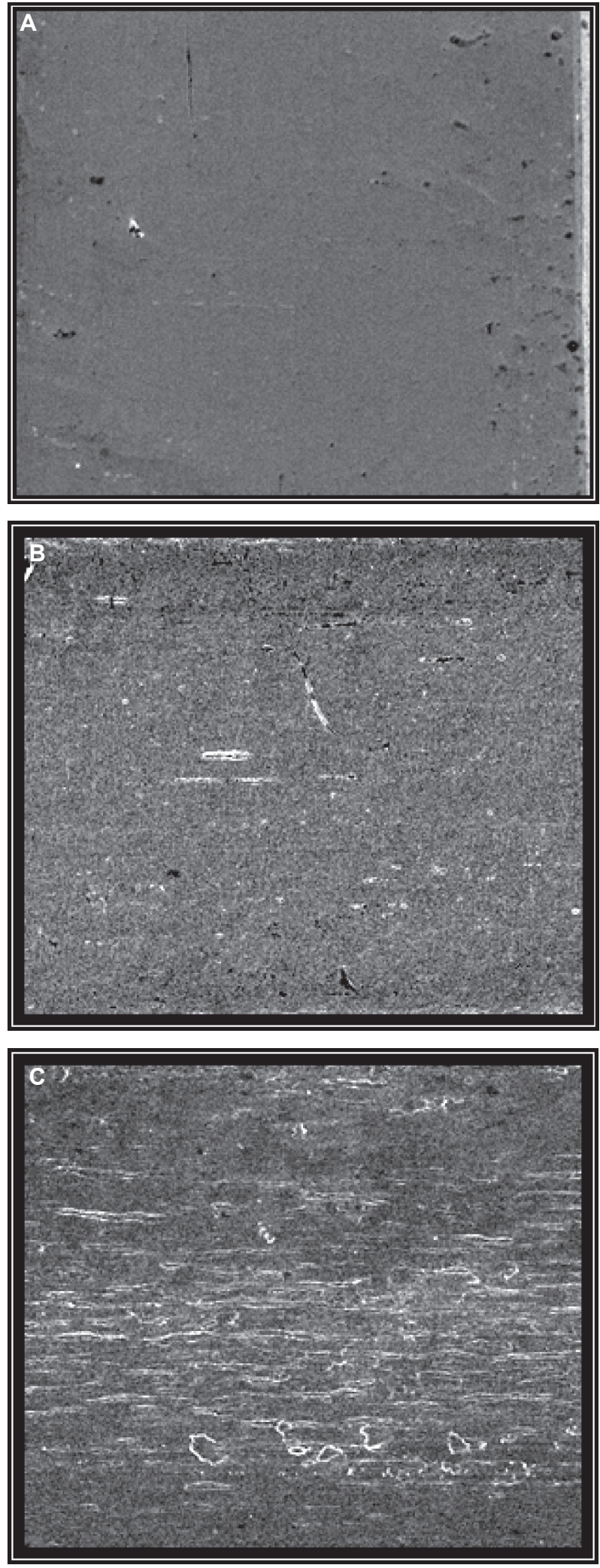

Figure 4- A) Stainless steel wire (200X); B) Nickeltitanium wire (200X); and C) Beta-titanium wire (200X) before mechanical testing

traction testing, combined with $0.019 " \times 0.025^{\prime \prime}$ stainless steel, nickel-titanium, and beta-titanium archwires at angulations of 0, 5, and 10 degrees.

Each bracket/wire combination was submitted to 
10 mechanical tests. This methodology was adopted because, under clinical conditions, the bracket shifts along the archwire or vice-versa repeatedly during the sliding mechanics for a given period of time ${ }^{21}$. Although some authors had reported that the repeated use of brackets and wires can cause surface alterations in these materials, thus changing the friction coefficient values produced ${ }^{16}$, the present study has demonstrated that no significant changes occurred in the friction force values regarding the use of one bracket during the mechanical tests repeated 10 times.

The brackets used had MBT prescription ( $17^{\circ}$ torque and $4^{\circ}$ angulation), and torque was eliminated by attaching the brackets to a metallic circular piece with the same 17-degree inclination. Angulation was eliminated with a 4-degree rotation in the metallic piece towards a counter-clockwise direction in relation to the angulation of the bracket slot so that the wires, which were attached by a fixed device, remained passive inside de slots. As the brackets had the same angulation and torque in the present study, the most important issue was to compare the frictional values found instead of quantifying the friction that occurs clinically in either material. Nevertheless, elimination of torque allows comparison with other brackets requiring different prescriptions.

In the present study, only static friction was evaluated as it is the force that must be overcome so that dental movement can be initiated. Moreover, static friction is greater than the kinetic one, since the latter is related only to the maintenance of movement ${ }^{2}$.

The large standard deviation observed in statistical descriptions was also found in previous studies that evaluated different combinations of brackets/wires ${ }^{4,9,18}$ and it can be explained by the mean maximum forces that refer to different brackets, wires and angles together with the heterogeneity inherent to each one.

Scanning electron microscopy showed the roughness increases in the following order: stainless steel, nickel-titanium, and beta-titanium (Figures $4 \mathrm{~A}, 4 \mathrm{~B}$, and 4C). Mechanical tests showed that the friction results followed the same order of roughness for different combinations of wires and conventional brackets, meaning that the surface roughness of the wire seems to influence the frictional values. Previous studies have also found low friction for stainless steel archwires, followed by nickel-titanium and beta-titanium ones ${ }^{14,21}$. However, the self-ligating brackets (Smartclip) exhibited lower friction force when combined with nickel-titanium, stainless steel, and beta-titanium wires, in this order. A possible explanation is the fact that Smartclip has nickel-titanium clips, which in contact with wires of the same material could produce lower frictional values. It was not previously found in the literature, since, to our knowledge, there are no studies evaluating the Smartclip brackets associated with different types of wire. Therefore, further researches should be performed in order to quantify this issue of yielding virtually null friction values.

With regard to the angulation between bracket and orthodontic wire, the results demonstrated that frictional values were statistically significant and directly proportional to the increase in angulation, both in conventional and self-ligating brackets, a finding also corroborated elsewhere ${ }^{2,25}$. Interestingly, the greater the angulation the lower the friction difference between self-ligating and conventional brackets. This probably occurs because there is a space between the wire and the slots of self-ligating brackets, at zero-degree angulation, thus yielding virtually null frictional values. On the other hand, the ligation force produced by conventional brackets causes the wire to be in contact with lateral walls of the slot, thus yielding binary forces that increase the friction. Moreover, the ligation force is not present in the self-ligating brackets.

Our results show that even at different angulations, the self-ligating brackets showed significantly lower friction force values than the conventional brackets, thus the self-ligating brackets can be an interesting option for the translation of a tooth or block of teeth using sliding mechanics. In clinical orthodontics, when the tooth begins to move the wires start to contact the corners of the bracket and binding (angulation) starts $^{3}$. Finite element analysis has shown that $60 \%$ to $80 \%$ of the applied orthodontic force is lost during retraction by sliding mechanics of a canine along a rectangular archwire ${ }^{19}$. Since the orthodontic force must overcome the frictional resistance, minimizing friction will result in reduced levels of the clinically applied force needed for moving the teeth. Such reduction might shorten the treatment period and improve anchorage control ${ }^{25}$. In addition, low forces will reduce the risk of root resorption ${ }^{11}$.

Low friction may also be desired during the orthodontic phase of alignment ${ }^{27}$, however, in the last phases, higher friction force can be used to obtain a three-dimensional control of the tooth position. A recent study evaluating how self-ligating brackets compared to conventional brackets in terms of orthodontists' perceptions showed that the first ones were preferred during the initial stage of treatment ${ }^{24}$, but practitioners preferred conventional brackets during the finishing stages. The solution could be a self-ligating bracket that can be ligated if necessary.

There seems to be agreement about the use of round wires for self-ligating brackets 
as such combination produces significantly lower friction compared to that of conventional brackets ${ }^{1,10,14,16,20,22,25}$. These results allow us to conclude that the same outcome occurs even in rectangular wires, which is in accordance with some previous studies ${ }^{5,6,9}$ but in discordance with other authors, who found no significant difference ${ }^{12,13,22,23,28}$ or observed greater friction in the self-ligating brackets ${ }^{25}$. These discrepancies can be attributed to differences between experimental models, including shapes, sizes and prescriptions of self-ligating brackets, as well as clips or caps made of different materials.

In vitro studies contribute to a better understanding of the frictional resistance in selfligating brackets, but they do not replicate what really occurs during clinical dental movement ${ }^{21}$. The frictional values found in the present study should be used as mean values for comparison of the effects of different types of brackets, archwires, and angulations instead of in vivo friction quantification. Investigation of frictional effetcs in vivo is needed so that bracket systems may be further refined to reduce friction and optimize sliding mechanics.

\section{CONCLUSIONS}

1. Self-ligating brackets showed significantly lower friction compared with conventional brackets, even with increased bracket/wire angulation;

2. Among the combinations tested, it was observed that self-ligating brackets (Smartclip) associated with nickel-titanium archwire produced the lowest friction.

\section{ACKNOWLEDGEMENTS}

The Authors wish to thank 3M/Unitek for providing the materials tested in this work.

\section{REFERENCES}

1- Baccetti T, Franchi L, Camporesi M, Defraia E, Barbato E. Forces produced by different nonconventional bracket or ligature systems during alignment of apically displaced teeth. Angle Orthod. 2009;79:533-9.

2- Benancon RM. The encyclopedia of physics. 3.ed. New York: Van Nostrand Reinhold; 1985.

3- Burrow SJ. Canine retraction rate with self-ligating brackets vs conventional edgewise brackets. Angle Orthod. 2010;80:438-45. 4- Cacciafesta V, Sfondrini MF, Ricciardi A, Scribante A, Klersy C, Auricchio $F$. Evaluation of friction of stainless steel and esthetic self-ligating brackets in various bracket-archwire combinations. Am J Orthod Dentofacial Orthop. 2003;124:395-402.

5- Ehsani S, Mandich MA, El-Bialy TH, Flores-Mir C. Frictional resistance in self-ligating orthodontic brackets and conventionally ligated brackets. Angle Orthod. 2009;79:592-601.

6- Franchi L, Baccetti T, Camporesi M, Barbato E. Forces released during sliding mechanics with passive self-ligating brackets or nonconventional elastomeric ligatures. Am J Orthod Dentofacial Orthop. 2008;133:87-90.
7- Gandini P, Orsi L, Bertoncini C, Massironi S, Franchi L. In vitro frictional forces generated by three different ligation methods. Angle Orthod. 2008;78:917-21.

8- Griffiths HS, Sherriff M, Ireland AJ. Resistance to slinding with 3 types of elastomeric modules. Am J Orthod Dentofacial Orthop. 2005;127:670-5.

9- Hain M, Dhopatkar A, Rock P. A comparison of different ligation methods on friction. Am J Orthod Dentofacial Orthop. 2006;130:666-70.

10- Harradine NW. Self-ligating brackets: where are we now? J Orthod. 2003;30:262-73.

11- Harry MR, Sims MR. Root resorption in bicuspid intrusion. A scanning electron microscope study. Angle Orthod.1982;52:235-58. 12- Heano SP, Kusy RP. Evaluations of the frictional resistance of conventional and self-ligating bracket designs using standardized archwires and dental typodonts. Angle Orthod. 2004;74:202-11. 13- Heano SP, Kusy RP. Frictional evaluations of dental typodont model using four self-ligating designs and a conventional design. Angle Orthod. 2005;75:75-85.

14- Ireland AJ, Sherriff M, McDonald F. Effect of bracket and wire composition on frictional forces. Eur J Orthod. 1991;13:322-8.

15- Kapila S, Angolkar PV, Duncanson MG Jr, Nanda RS. Evaluation of friction between edgewise stainless steel brackets and orthodontic wires of four alloys. Am J Orthod Dentofacial Orthop. 1990;98:117-26.

16- Kapur R, Sinha PK, Nanda RS. Comparison of frictional resistance in titanium and stainless steel brackets. Am J Orthod Dentofacial Orthop. 1999;116:271-4.

17- Khambay YB, Millet D, Mchung S. Evaluation of methods of archwire ligation on frictional resistance. Eur J Orthod. 2004;26:327-32.

18- Kim TK, Kim KD, Baek SH. Comparison of frictional forces during the initial leveling stage in various combinations of selfligating brackets and archwires with a custom-designed typodont system. Am J Orthod Dentofacial Orthop. 2008;133:187.e15-24. 19- Kojima Y, Fukui $\mathrm{H}$. Numerical simulation of canine retraction by sliding mechanics. Am J Orthod Dentofacial Orthop. 2005; 127:542-51.

20- Muguruma T, Iijima M, Brantley WA, Mizoguchi I. Effects of a diamond-like carbon coating on the frictional properties of orthodontic wires. Angle Orthod. 2011;81:141-8.

21- Nishio C, Motta AF, Elias CN, Mucha JN. In vitro evaluation of frictional forces between archwires and ceramic brackets. Am J Orthod Dentofacial Orthop. 2004;125:56-64.

22- Pliska BT, Beyer JP, Larson BE. A comparison of resistance to sliding of self-ligating brackets under an increasing applied moment. Angle Orthod. 2011;81:794-9.

23- Pliska BT, Fuchs RW, Beyer JP, Larson BE. Effect of applied moment on resistance to sliding among esthetic self-ligating brackets. Angle Orthod. 2014;84:134-9.

24- Prettyman C, Best AM, Lindauer SJ, Tufekci E. Self-ligating vs conventional brackets as perceived by orthodontists. Angle Orthod. 2012;82:1060-6.

25- Readlich M, Mayer Y, Harari D, Lewinstein I. In vitro study of frictional forces during sliding mechanics of "reduced-friction" brackets. Am J Orthod Dentofacial Orthop. 2003;124:69-73.

26- Rinchuse DJ, Miles PG. Self-ligating brackets: present and future. Am J Orthod Dentofacial Orthop. 2007;132:16-22.

27- Tecco S, Festa F, Caputi S, Traini T, Di Iorio D, D'Attilio M. Friction of convencional and self-ligating brackets using a 10 bracket model. Angle Orthod. 2005;75:1041-5.

28- Tecco S, Di Iorio D, Cordasco G, Verrochi I, Festa F. An in vitro investigation of the influence of self-ligating brackets, low friction ligatures, and archwire on frictional resistance. Eur J Orthod. 2007;29:390-7. 